\title{
Two-dimensional equilibrium island shape and step free energies of $\mathrm{Cu}(001)$
}

\author{
Ronny Van Moere, Harold J. W. Zandvliet, and Bene Poelsema \\ Solid State Physics Group and MESA + Research Institute, University of Twente, P.O. Box 217, 7500 AE Enschede, The Netherlands
}

(Received 3 December 2002; revised manuscript received 10 March 2003; published 30 May 2003)

\begin{abstract}
We have derived expressions for the free energies of the densely packed [110] and 100\% kinked [010] step edges of $\mathrm{Cu}(001)$, including both meandering and vibrational entropy terms. The meandering entropy is calculated by taking into account nearest-neighbor and next-nearest-neighbor interactions between the $\mathrm{Cu}$ atoms. The vibrational entropy is determined within the framework of an isotropic Einstein oscillator. By using the earlier obtained kink creation energy by Giesen, Steimer, and Ibach [Surf. Sci. 471, 80 (2001)] and taking the strength of the next-nearest-neighbor interaction as the only fitting parameter, we obtain perfect agreement between the ratio of the calculated and experimentally determined ratio of the step free energies (i.e., $\left.F_{[010]} / F_{[110]}\right)$. Moreover, in contrast to the Ising model we predict that the two-dimensional equilibrium $\mathrm{Cu}(001)$ island shape at $T=0 \mathrm{~K}$ is not a perfect square.
\end{abstract}

DOI: 10.1103/PhysRevB.67.193407

PACS number(s): 68.35.Md, 68.35.Bs

Steps play an important role in many equilibrium and nonequilibrium surface processes, such as thermal roughening, Ostwald ripening, crystal growth, and etching. The free energy of steps also controls the relative size of the facets in the equilibrium shape of crystals and the curvature of rough surfaces. With this in mind, it is self-evident that knowledge of step energies is a prerequisite for a quantitative description of these surface processes. The experimental determination of a reliable value of the step free energy is, however, far from trivial. For instance, the step free energy derived from the chemical potentials of islands observed in Ostwald ripening of islands is found to be too high, for reasons not yet understood. ${ }^{1}$

About a decade ago Swartzentruber et al. ${ }^{2}$ showed in an elegant way that the step formation energies of $\mathrm{Si}(001)$ can be extracted from a detailed analysis of the step-edge roughness. These authors used Boltzmann statistics for independent kink excitations and a simple solid-on-solid model including only nearest-neighbor interactions to determine the kink and step formation energies of $\mathrm{Si}(001)$. This method can be extended to include next-nearest-neighbor interactions as well $^{3}$ and is in principle also applicable to other surfaces. However, the applicability is more satisfactory for materials with covalent bonds, such as semiconductors, than for metals. ${ }^{4}$ Besides the determination of the kink and step-edge formation energies from step-edge roughness, other routes have been explored as well. ${ }^{5-14}$ For instance, Schlösser et $a l .{ }^{6}$ put forward the idea to extract the absolute value of the mean step energy from temporal equilibrium shape fluctuations of an island. Schulze Icking-Konert et al. ${ }^{7}$ used the temperature dependence of the equilibrium shape of islands to determine the step energies. More recently, Giesen et al. ${ }^{8}$ introduced a novel method denoted as the minimum curvature model to derive the step energies.

In this Brief Report we extend the model introduced by Schulze Icking-Konert et al. ${ }^{7}$ by including next-nearestneighbor interactions between the $\mathrm{Cu}$ atoms as well. By including these next-nearest-neighbor interactions the equilibrium shape of $\mathrm{Cu}$ (vacancy) islands on $\mathrm{Cu}(001)$ at $T=0 \mathrm{~K}$ is no longer an exact square (i.e., the ratio of the step-edge formation energies, $E_{[010]} / E_{[110]}$, is in general $<\sqrt{2}$ ). If we assume that the next-nearest-neighbor interaction is attractive, it will increase the formation free energy of a [110] step more than that of a $[010]$ step. The ratio $E_{[010]} / E_{[110]}$ will therefore become smaller than $\sqrt{2}$. By taking the kink energy of a [110] step on $\mathrm{Cu}(001)$ (Ref. 8) and tuning only the next-nearest-neighbor interaction energy, a perfect fit between the existing experimental data of the equilibrium shape of islands on $\mathrm{Cu}(001)$ and our model can be obtained. Finally, in order to obtain an accurate temperature dependence of the step free energies we have also included the additional vibrational entropy of the step-edge atoms.

The step free energy per unit length and per unit step height, $F(T)$, is defined by

$$
F(T)=-k T \ln Z=-k T \ln \left(\sum_{j} e^{-E_{j} / k T}\right),
$$

where the summation runs over all possible step configurations and $E_{j}$ refers to the formation energy of the $j$ th configuration, $Z$ to the partition function, $T$ to the temperature, and $k$ to Boltzmann's constant. In Fig. 1 a schematic diagram of the $\mathrm{Cu}$ adatom island on a $\mathrm{Cu}(001)$ is depicted. The densely packed step edges run along the $\langle 110\rangle$ directions, whereas the $100 \%$ kinked step edges run along the $\langle 010\rangle$ directions. If only nearest-neighbor $\left(\varepsilon_{1}\right)$ and next-nearestneighbor $\left(\varepsilon_{2}\right)$ interactions are considered [see Fig. 2(a)], the partition function $Z$ of a [110] step on a $\mathrm{Cu}(001)$ surface is given by ${ }^{4}$

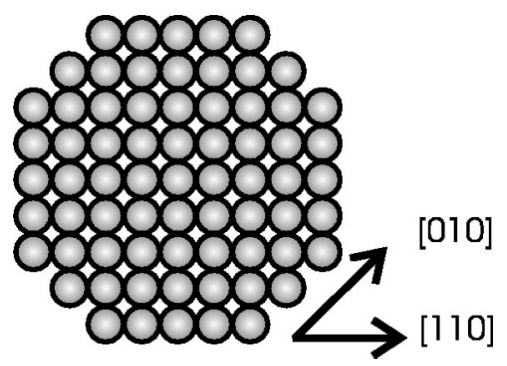

FIG. 1. Schematic diagram of a $\mathrm{Cu}$ adatom island on a $\mathrm{Cu}(001)$ surface. Both [110] and [010] directions are outlined. 


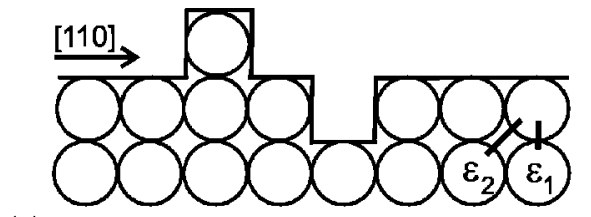

(a)

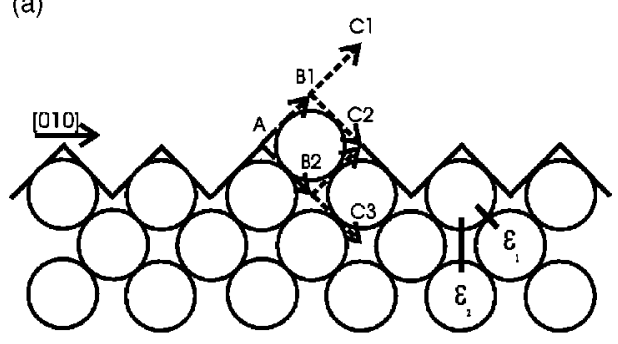

(b)

FIG. 2. (a) Schematic diagram of a [110] step edge on $\mathrm{Cu}(001)$. The nearest-neighbor $\left(\varepsilon_{1}\right)$ and next-nearest-neighbor $\left(\varepsilon_{2}\right)$ interaction energies are indicated. (b) Schematic diagram of a [010] step edge on $\mathrm{Cu}(001)$. The nearest-neighbor $\left(\varepsilon_{1}\right)$ and next-nearestneighbor $\left(\varepsilon_{2}\right)$ interaction energies are indicated. The dashed lines $\mathrm{AB} 1$ and $\mathrm{AB} 2$ refer to the two energetically most favorable pathways. The outlined pathways have the same step-edge length: however, pathway $\mathrm{AB} 2$ has a corner, whereas pathway $\mathrm{AB} 1$ has not. At point $\mathrm{B} 1$ the pathway can go either in the $\mathrm{C} 1$ or $\mathrm{C} 2$ direction. At point $\mathrm{B} 2$ the pathway can go either in the $\mathrm{C} 2$ or $\mathrm{C} 3$ direction.

$$
Z_{[110]}=e^{-\left(\varepsilon_{1} / 2+\varepsilon_{2}\right) / k T}\left(1+2 \sum_{n=1}^{\infty} e^{-\left[n \varepsilon_{1} / 2+(n-1) \varepsilon_{2}\right] / k T}\right) .
$$

The factor of 2 in Eq. (2) arises from the fact that both negative and positive kinks are considered. Substituting the partition function $Z$ into Eq. (1) results in ${ }^{4}$

$$
F_{[110]}(T)=\left(\frac{\varepsilon_{1}}{2}+\varepsilon_{2}\right)-k T \ln \left(1+\frac{2 e^{-\varepsilon_{1} / 2 k T}}{\left(1-e^{-\left(\varepsilon_{1} / 2+\varepsilon_{2}\right) / k T}\right)}\right) .
$$

In order to find an expression for the step free energy of $100 \%$ kinked [010] step we follow a similar procedure. We take into account only the two energetically most favorable pathways, ${ }^{7}$ because all the other pathways involve significantly more step edge length and are therefore much higher in energy [see Fig. 2(b)]. The step-edge energies per unit length $\frac{1}{2} \sqrt{2} a$ (measured in the [010] direction) are then $\varepsilon_{1} / 2+\varepsilon_{2} / 2$ and $\varepsilon_{1} / 2+\varepsilon_{2}$, respectively. The partition function of a $[010]$ step is given by

$$
Z_{[010]}=e^{-\left[\left(\varepsilon_{1}+\varepsilon_{2}\right) / 2\right] / k T}+e^{-\left(\varepsilon_{1} / 2+\varepsilon_{2}\right) / k T} .
$$

The free energy of a [010] step per unit length $a$ ( $a$ $=2.55 \AA$ is the surface lattice constant) is then represented by

$$
F_{[010]}(T)=\sqrt{2}\left[\left(\frac{\varepsilon_{1}+\varepsilon_{2}}{2}\right)-k T \ln \left(1+e^{-\varepsilon_{2} / 2 k T}\right)\right] .
$$

The partition function $Z$ considered in Eq. (4) considers a line segment of length $\frac{1}{2} \sqrt{2} a$ in the [010] direction and therefore the factor of $\sqrt{2}$ is needed in Eq. (5) in order to convert the step free energy to a free energy per unit length $a$ in the [010] direction. For a vanishing next-nearest-neighbor interaction-i.e., $\varepsilon_{2}=0-$ Eq. (5) reduces, as it should, to the expression derived earlier by Schulze Icking-Konert et al., ${ }^{7}$ i.e.,

$$
F_{[010]}(T)=\sqrt{2}\left[\left(\frac{\varepsilon_{1}}{2}\right)-k T \ln 2\right]
$$

So far, we have included only the meandering entropy. As pointed out by Frenken and Stoltze, ${ }^{15}$ Schulze Icking-Konert et al., ${ }^{7}$ and Bonzel and Edmundts ${ }^{16}$ the vibrational entropy terms contribute to the step free energy as well. Here we include only the vibrational entropy terms of the step atoms to the step free energy, whereas higher-order contributions of the vibrational entropy due to thermal kink generation are ignored. ${ }^{17}$ This approximation is appropriate as long as the temperature is below $\sim 0.5 T_{m}$ ( $T_{m}$ is the melting point). This holds for all data analyzed here below.

The free energy of an isotropic Einstein oscillator is given by

$$
F_{\mathrm{vib}}(T)=\frac{3}{2} \hbar \omega+3 k T \ln \left(1-e^{-\hbar \omega / k T}\right)
$$

The factor of 3 accounts for the three orthogonal directions of vibration. The additional vibrational free energy of a step atom with respect to a terrace atom is then

$$
\Delta F_{\text {step }}(T)=\frac{3}{2} k\left(\Theta_{\text {step }}-\Theta_{\text {ter }}\right)+3 k T \ln \left(\frac{1-e^{-\Theta_{\text {step }} / T}}{1-e^{-\Theta_{\text {ter }} / T}}\right),
$$

where $\Theta_{\text {ter,step }}\left(\equiv \hbar \omega_{\text {ter,step }} / k\right)$ is the Debye temperature of a terrace (step) atom. The bulk Debye temperature of $\mathrm{Cu}$ is $343 \mathrm{~K}$. As a crude estimate, we have related the relative Debye temperatures of terrace, [110], and [010] step atoms to the number of nearest-neighbor bonds: i.e., $\Theta_{\text {ter }}$ $=\Theta_{\text {bulk }} \sqrt{\frac{8}{12}}, \Theta_{[110]}=\Theta_{\text {bulk }} \sqrt{\frac{7}{12}}$, and $\Theta_{[010]}=\Theta_{\text {bulk }} \sqrt{\frac{6}{12}}$. If both meandering and vibrational entropy terms are included, the step free energies of the [110] and [010] steps are given by, respectively,

$$
\begin{aligned}
F_{[110]}(T)= & \left(\frac{\varepsilon_{1}}{2}+\varepsilon_{2}\right)-k T \ln \left(1+\frac{2 e^{-\varepsilon_{1} / 2 k T}}{\left(1-e^{-\left(\varepsilon_{1} / 2+\varepsilon_{2}\right) / k T}\right)}\right) \\
& +\left[\frac{3}{2} k\left(\Theta_{[110]}-\Theta_{\text {ter }}\right)+3 k T \ln \left(\frac{1-e^{-\Theta_{[110]} / T}}{1-e^{-\Theta_{\text {ter }} / T}}\right)\right],
\end{aligned}
$$

$$
\begin{aligned}
F_{[010]}(T)= & \sqrt{2}\left[\left(\frac{\varepsilon_{1}+\varepsilon_{2}}{2}\right)-k T \ln \left(1+e^{-\varepsilon_{2} / 2 k T}\right)\right] \\
& +\frac{1}{2} \sqrt{2}\left[\frac{3}{2} k\left(\Theta_{[010]}-\Theta_{\mathrm{ter}}\right)\right. \\
& \left.+3 k T \ln \left(\frac{1-e^{-\Theta_{[010]} / T}}{1-e^{-\Theta_{\mathrm{ter}} / T}}\right)\right] .
\end{aligned}
$$




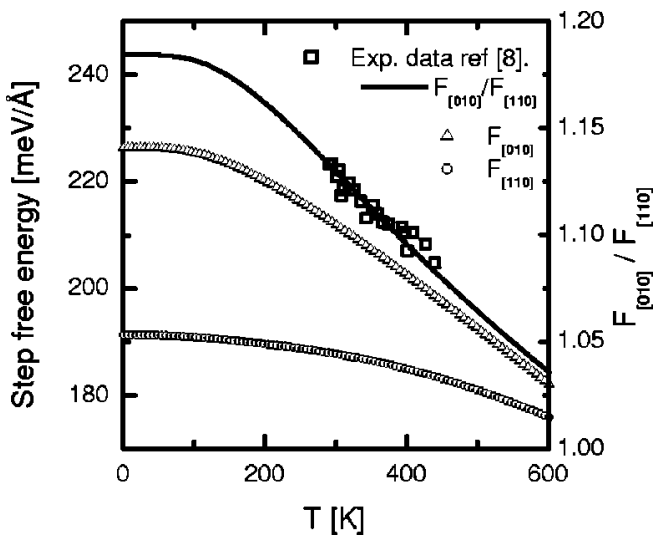

FIG. 3. Plot of the step free energies of [110] (circles) and [010] (triangles) steps of a $\mathrm{Cu}(001)$ surface as a function of the temperature $\left(\varepsilon_{1}=262 \mathrm{meV}\right.$ and $\left.\varepsilon_{2}=63 \mathrm{meV}\right)$. The solid line refers to the calculated ratio of the step free energies $\left(F_{[010]} / F_{[110]}\right)$. The squares represent the experimentally determined ratio of the step free energies and are taken from Ref. 8.

The step edge formation energies $E_{[110],[010]}$ can be found by inserting $T=0 \mathrm{~K}$ in Eqs. (8):

$$
\begin{gathered}
E_{[110]}=\left(\frac{\varepsilon_{1}}{2}+\varepsilon_{2}\right)+\frac{3}{2} k\left(\Theta_{[110]}-\Theta_{\text {ter }}\right), \\
E_{[010]}=\left(\frac{1}{2} \sqrt{2} \varepsilon_{1}+\frac{1}{2} \sqrt{2} \varepsilon_{2}\right)+\frac{3 \sqrt{2}}{4} k\left(\Theta_{[010]}-\Theta_{\text {ter }}\right) .
\end{gathered}
$$

The kink formation energy $\varepsilon_{\text {kink }}$ for a kink in a [110] step is the step-edge formation energy minus the next-nearestneighbor interaction energy:

$$
\varepsilon_{\text {kink }}=\frac{\varepsilon_{1}}{2}+\frac{3}{2} k\left(\Theta_{[110]}-\Theta_{\text {ter }}\right) .
$$

The vibrational entropy part of the step free energies requires only the bulk Debye temperature of $\mathrm{Cu}$ as an input $(\Theta$ $=343 \mathrm{~K})$. Because the kink energy of a [110] step of $\mathrm{Cu}(001)$ is determined by several independent methods, giving values in the range from $128 \mathrm{meV} / a$ (spatial step correlation $^{18}$ ) to $131 \mathrm{meV} / \mathrm{a}$ (minimum curvature model ${ }^{8}$ ), we have taken a value of $\varepsilon_{1}=262 \mathrm{meV}$ (the kink energy then varies from $131 \mathrm{meV} / a$ if the zero-point vibrational energy is not included to $128 \mathrm{meV} / \mathrm{a}$ if the zero-point vibrational energy is included). In Fig. 3 plots of the step free energies versus the temperature are shown. An excellent fit between the ratio of the step free energies and the experimental de- termined temperature-dependent equilibrium shape of islands on $\mathrm{Cu}(001)$ can be obtained if one takes $\varepsilon_{2}$ $=63 \mathrm{meV} /$ corner (see Fig. 3). One then finds for the stepedge formation energies $E_{[110]}=193 \mathrm{meV} / a$ and $E_{[010]}$ $=227 \mathrm{meV} / a$, respectively. The value of our step formation energy of [110] step is slightly lower than the $220 \mathrm{meV} / a$ as reported by Giesen et al. ${ }^{8}$ as well as Steimer et al. ${ }^{9}$ The agreement between our value and recent tight-binding calculations of Raouafi et al. ${ }^{19}(191 \mathrm{meV} / \mathrm{a})$ is excellent. However, the next-nearest-neighbor interaction we found $\left(\varepsilon_{2} / 2\right.$ $=31 \mathrm{meV}$ ) is substantially larger than the value obtained by calculations of Raouafi et al. ${ }^{19}(13 \mathrm{meV})$ and of Vitos et al. ${ }^{20}$ $(18 \mathrm{meV})$. It should be noted, however, that the vibrational free energy contributes only about a few meV/ $a$ at low temperatures to about $10-15 \mathrm{meV} / a$ at temperatures around 400 $\mathrm{K}$. The ratio of the step-edge formation energies-i.e., $F_{[010]}(T=0) / F_{[110]}(T=0)$-is about 1.18 , which is much closer to the experimentally extrapolated ratio of 1.24 (Ref. 8) than the $\sqrt{2}$ as predicted by the Ising model. ${ }^{21-23}$

It should be pointed out here that the equilibrium shape of islands or vacancy islands might depend on the surface stress as well. ${ }^{24,25}$ However, the $\mathrm{Cu}(001)$ surface is isotropic and therefore the surface stress is isotropic too. For anisotropic surfaces, such as, for instance, $\mathrm{Si}(001)$ and $\mathrm{Ge}(001)$, a force monopole is present at the boundaries of the islands, giving rise to an additional strain energy term. ${ }^{25-27}$ For island sizes below a critical island size the island-edge free energy is dominated by the step-edge free energies, whereas strain energy becomes the leading free-energy term beyond this critical island size. Free-energy minimization results in a minimization of the total island-edge length below the critical island size (resulting in compact islands) and in a maximization of the total island-edge length beyond the critical island size (resulting in elongated islands).

In summary, the free energies of $\mathrm{Cu}(001)$ steps have been derived including both meandering and vibrational entropy terms. We show that the addition of next-nearest-neighbor interactions is essential in order to obtain a good fit between the ratio of the step free energies and temperature-dependent equilibrium island shape. The next-nearest-neighbor interaction energy is attractive and about 4 times weaker than the nearest-neighbor interaction energy. Despite the fact that the next-nearest-neighbor interaction energy is substantially lower than the nearest-neighbor interaction, it properly describes the temperature dependence of the equilibrium shape of $\mathrm{Cu}$ adatom and vacancy islands on $\mathrm{Cu}(001)$.

This work is part of the research program of the "Stichting voor Fundamenteel Onderzoek der Materie" (FOM).
${ }^{1}$ G. S. Icking-Konert, M. Giesen, and H. Ibach, Surf. Sci. 398, 37 (1998).

${ }^{2}$ B. S. Swartzentruber, Y.-W. Mo, R. Kariotis, M. G. Lagally, and M. B. Webb, Phys. Rev. Lett. 65, 1913 (1990).

${ }^{3}$ H. J. W. Zandvliet, H. B. Elswijk, E. J. van Loenen, and D. Dijkkamp, Phys. Rev. B 45, 5965 (1992).
${ }^{4}$ H. J. W. Zandvliet, Rev. Mod. Phys. 72, 593 (2000).

${ }^{5}$ N. C. Bartelt, R. M. Tromp, and E. D. Williams, Phys. Rev. Lett. 73, 1656 (1994).

${ }^{6}$ D. C. Schlösser, L. K. Verheij, G. Rosenfeld, and G. Comsa, Phys. Rev. Lett. 82, 3843 (1999).

${ }^{7}$ G. Schulze Icking-Konert, M. Giesen, and H. Ibach, Phys. Rev. 
Lett. 83, 3880 (1999).

${ }^{8}$ M. Giesen, C. Steimer, and H. Ibach, Surf. Sci. 471, 80 (2001).

${ }^{9}$ C. Steimer, M. Giesen, L. Verheij, and H. Ibach, Phys. Rev. B 64, 085416 (2001).

${ }^{10}$ S. Kodambaka et al., Phys. Rev. Lett. 88, 146101 (2002).

${ }^{11}$ S. Kodambaka et al., Phys. Rev. B 67, 035409 (2003).

${ }^{12}$ S. V. Khare et al., Surf. Sci. 522, 75 (2003).

${ }^{13}$ S. Kodambaka et al., Surf. Sci. 513, 468 (2002).

${ }^{14}$ S. B. Albada, M. J. Rost, and J. W. M. Frenken, Phys. Rev. B 65, 205421 (2002).

${ }^{15}$ J. W. M. Frenken and P. Stoltze, Phys. Rev. Lett. 82, 3500 (1999).

${ }^{16}$ H. P. Bonzel and A. Emundts, Phys. Rev. Lett. 84, 5804 (2000).

${ }^{17}$ H. J. W. Zandvliet, O. Gurlu, and B. Poelsema, Phys. Rev. B 64, 073402 (2001).
${ }^{18}$ M. Giesen-Seibert, F. Schmitz, R. Jentjens, and H. Ibach, Surf. Sci. 329, 47 (1995).

${ }^{19}$ F. Raouafi, C. Barreteau, M. C. Desjonquères, and D. Spanjaard, Surf. Sci. 505, 183 (2002).

${ }^{20}$ L. Vitos, H. L. Skriver, and J. Kollar, Surf. Sci. 425, 212 (1999).

${ }^{21}$ C. Rottman and M. Wortis, Phys. Rev. B 24, 6274 (1981).

${ }^{22}$ R. K. P. Zia and J. E. Avron, Phys. Rev. B 25, 2042 (1982).

${ }^{23}$ R. K. P. Zia, J. Stat. Phys. 45, 801 (1986).

${ }^{24}$ J. Tersoff and R. M. Tromp, Phys. Rev. Lett. 70, 2782 (1993).

${ }^{25}$ A. Li, F. Liu, and M. G. Lagally, Phys. Rev. Lett. 85, 1922 (2000).

${ }^{26}$ M. T. Middel, H. J. W. Zandvliet, and B. Poelsema, Phys. Rev. Lett. 88, 196105 (2002).

${ }^{27}$ H. J. W. Zandvliet, Mod. Phys. Lett. B 11, 47 (1997). 